\title{
Carbon electrodes with ionophobic characteristics in organic electrolyte for high-performance electric double-layer capacitors
}

\author{
Xue Yin ${ }^{1}$, Jianqi Zhang ${ }^{2}$, Le Yang ${ }^{1}$, Wende Xiao ${ }^{3}$, Lei Zhou ${ }^{1}$, Yujing Tang ${ }^{4}$ and Wen Yang ${ }^{*}$
}

\begin{abstract}
Activated carbon (AC) in organic electrolytebased electric double-layer capacitors (EDLCs) usually suffers from low specific capacitance. Most studies on AC focus on improving its surface area and optimizing pore structures to enhance its electrochemical performance in EDLCs. Unfortunately, the interfacial microenvironment, which is composed of nanoporous carbon and the organic electrolyte confined in it, is always ignored. Herein, a simple and powerful strategy to create AC with an ionophobic surface is proposed to address the poor efficiency of the electric doublelayer process. The polar $\mathrm{C}-\mathrm{F}$ bonds formed in the $\mathrm{AC}$ material are characterized through near-edge $\mathrm{X}$-ray absorption fine structure and $\mathrm{X}$-ray photoelectron spectroscopy. The ionophobic characteristic of YP-F60s in an organic electrolyte is extensively studied via contact angle measurements and smallangle X-ray scattering spectroscopy. An EDLC constructed with YP-F60s as the electrode and $1 \mathrm{~mol} \mathrm{~L}^{-1}$ tetraethylammonium tetrafluoroborate/propylene carbonate as the electrolyte demonstrates high specific capacitance, low internal resistance, and excellent cycling stability. Our results successfully demonstrate the importance of the interfacial microenvironment of $\mathrm{AC}$ and its confined electrolyte to the electrochemical performance of EDLCs. Our work also offers new perspectives on the use of the $\mathrm{CF}_{4}$ plasma technique to fabricate low-cost superior carbon for EDLCs.
\end{abstract}

Keywords: nanoporous carbon, ionophobic characteristic, organic electrolyte, electric double-layer capacitors

\section{INTRODUCTION}

Electric double-layer capacitors (EDLCs) are alternative energy storage devices with high power densities, fast charge/discharge rates, and long cycling lives. Activated carbon (AC) is widely used in EDLCs as an electrode material on account of its large specific surface area and good electrical conductivity [1-5]. The charge/discharge process can be achieved within a short period using AC, but the energy density of the material is not satisfactory [6-8]. Eliad et al. $[9,10]$ found that AC nanostructures with nanopores (i.e., pore sizes equal to the diameter of a single desolvation ion) have greater capacitance compared with ordinary mesoporous AC materials. Therefore, extensive research efforts have been conducted to obtain different nanoporous AC materials to achieve maximum energy density and determine an optimal storage mechanism [11,12]. However, a comprehensive understanding of the dynamics between $\mathrm{AC}$ and its confined electrolyte is lacking.

The interface between the electrolyte and carbon structure is crucial for the charging mechanism. Grey and his co-workers [13] demonstrated that the ions confined in nanoporous carbon decrease the effective ions self-diffusion coefficient by over two orders of magnitude when compared with neat electrolytes. This finding indicates that the affinity of pores toward ions, i.e., their ionophilicity, reduces the charge storage efficiency. Kondrat et al. $[14,15]$ proposed that an unfavorable thermodynamic condition for ions inside nanopores facilitates the energy storage. The absence of ions in nanopores without an applied voltage is beneficial to ion charging and thus, could achieve superior energy capacity. Therefore, the ability to modify the interfacial microenvironment of $\mathrm{AC}$ and its confined electrolyte, is of great significance for understanding the mechanism of EDLCs and designing advanced electrode materials to enhance the adsorption/desorption capacity of electrolyte ions in carbon nanopores.

The surface chemistry of electrode materials can be tuned by using fluorinated porous silicon particles to modulate their interaction with electrolyte ions, thereby creating a favorable environment that would allow minimum electrolyte ion infiltration while maintaining maximum performance [16]. However, the synthesis and application of fluorinated carbon materials that could be used to alter the interfacial microenvironment of carbon electrodes in EDLCs have yet to be reported. Given this background, we propose a simple and effective fluorination treatment strategy to construct a carbon material with ionophobic characteristics and regulate the interfacial microenvironment to deepen the understanding of the mechanism of EDLCs.

Nanoporous carbon (YP-50) with ionophobic characteristics is obtained by $\mathrm{CF}_{4}$ plasma treatment to boost the electrochemical performance of EDLCs. The surface properties of YP50 are smoothly altered during plasma treatment in a suitable

\footnotetext{
${ }^{1}$ Key Laboratory of Cluster Science of Ministry of Education, Beijing Key Laboratory of Photoelectronic/Electrophotonic Conversion Materials, School of Chemistry and Chemical Engineering, Beijing Institute of Technology, Beijing 100081, China

${ }^{2}$ CAS Key Laboratory of Nanosystem and Hierarchical Fabrication, CAS Center for Excellence in Nanoscience, National Center for Nanoscience and Technology, Beijing 100190, China

${ }^{3}$ Key Laboratory of Advanced Optoelectronic Quantum Architecture and Measurement, Ministry of Education, School of Physics, Beijing Institute of Technology, Beijing 100081, China

${ }^{4}$ SINOPEC Beijing Research Institute of Chemical Industry, Beijing 100013, China

* Corresponding author (email: wenyang@bit.edu.cn)
} 
atmosphere. Fluorine (F) atoms on the surface of the nanoporous carbon form polar $\mathrm{C}-\mathrm{F}$ bonds, which decrease the affinity of the carbon materials to ions in the organic electrolyte. Thus, the interfacial microenvironment of the nanoporous carbon and its confined electrolyte can easily be tuned, resulting in improvements in the EDLC process, low internal resistance, and superior cycle stability.

\section{EXPERIMENTAL SECTION}

\section{Materials}

YP-50, commercially available biochar obtained from Kuraray Company, was synthesized from coconut and initially activated by steam. All reagents, including conductive carbon black (Super P), polyvinylidene fluoride (PVDF), and $N$-methyl pyrrolidone, were obtained from Alfa Aesar.

\section{$\mathrm{CF}_{4}$ plasma treatment of the electrode materials}

YP-50 was treated with plasma in $\mathrm{CF}_{4}$ gas. The plasma system included a quartz tube with a vacuum pump, a radio frequency source, gas supplies, and the carbon sample to be treated inside the tube. Plasma activation was performed in a quartz tube connected to gas cylinders with a radio frequency and power of $13.56 \mathrm{MHz}$ and $60 \mathrm{~W}$, respectively. The reactive gas flow rate was controlled by a needle valve to maintain a discharge pressure of $\sim 0.2$ Torr $(1$ Torr $=133.3 \mathrm{~Pa})$, and the activation time ranged from 30 to $90 \mathrm{~s}$. The resultant samples (YP-F) were denoted as YP-F30s, YP-F60s, and YP-F90s.

\section{Material characterization}

The morphologies of all samples were characterized using a scanning electron microscope (SEM). Transmission electron microscopy (TEM) and high-resolution TEM (HRTEM) were used to observe the microstructures of all samples. BrunauerEmmett-Teller tests were conducted on a Micromeritics ASAP 2020 system to obtain the specific surface area and pore-size distribution of the samples. Raman spectroscopy (NRS-5100; excitation wavelength, $532 \mathrm{~nm}$ ) was used to characterize the structure of the biochar at $25^{\circ} \mathrm{C}$. Structural analysis of the samples was performed by powder X-ray diffraction (XRD, D8 ADVANCE, Germany). The elemental composition and che- mical status of the product surfaces were analyzed using X-ray photoelectron spectroscopy (XPS) and near-edge X-ray absorption fine structure (NEXAFS) spectroscopy. Contact angles (CAs) were measured by an OCA 20 contact angle system (Dataphysics, Germany) using the organic electrolyte as the test liquid. The ion adsorption of the porous carbon electrode in the organic electrolyte was determined via small-angle X-ray scattering spectroscopy (SAXS, Nanostar, Bruker AXS).

\section{Assembly of the supercapacitors}

The working electrode of the supercapacitors was prepared by coating a mixture of $80 \mathrm{wt} \%$ active material, $10 \mathrm{wt} \%$ Super P, and $10 \mathrm{wt} \%$ PVDF binder on an $\mathrm{Al}$ foil current collector. The obtained working electrode was dried under vacuum at $80^{\circ} \mathrm{C}$ for $24 \mathrm{~h}$. After drying, the coated $\mathrm{Al}$ foil was sheared into round plates measuring $0.94985 \mathrm{~cm}^{2}$ in area and weighed. The loading mass of the electrode on each $\mathrm{Al}$ plate was approximately $2 \mathrm{mg}$. Finally, the two symmetrical electrodes were assembled into a CR2032 coin cell using cellulose paper as the separator and $1 \mathrm{~mol} \mathrm{~L}^{-1}$ tetraethylammonium tetrafluoroborate (TEATFB)/ propylene carbonate (PC) as the electrolyte.

\section{Electrochemical measurements}

A CHI 760D electrochemistry workstation was used for cyclic voltammetry (CV). Galvanostatic charge/discharge (GCD) cycles were recorded using a Land Cell tester with a working voltage range of $0-2.7 \mathrm{~V}$. Electrical impedance spectroscopy (EIS) was performed using a $\mathrm{CHI} 760 \mathrm{D}$ electrochemical workstation with an alternating current (AC) amplitude of $\pm 5 \mathrm{mV}$ and a frequency range of $100 \mathrm{kHz}-0.1 \mathrm{~Hz}$. All tests were carried out at a constant temperature of $25^{\circ} \mathrm{C}$.

\section{RESULTS AND DISCUSSION}

Nanoporous YP-50 with ionophobic characteristics was fabricated, as shown in Scheme 1, by linking F atoms to the carbon surface. The microstructures and morphologies of the samples were observed by SEM and TEM. After $\mathrm{CF}_{4}$ plasma treatment, all samples retain the bulk morphology and particle distribution of the original YP-50 (Fig. S1). The TEM images reveal that the morphology of YP-F60s is nearly unchanged compared with that of YP-50 (Fig. 1a, b). Thus, $\mathrm{CF}_{4}$ plasma treatment may hardly

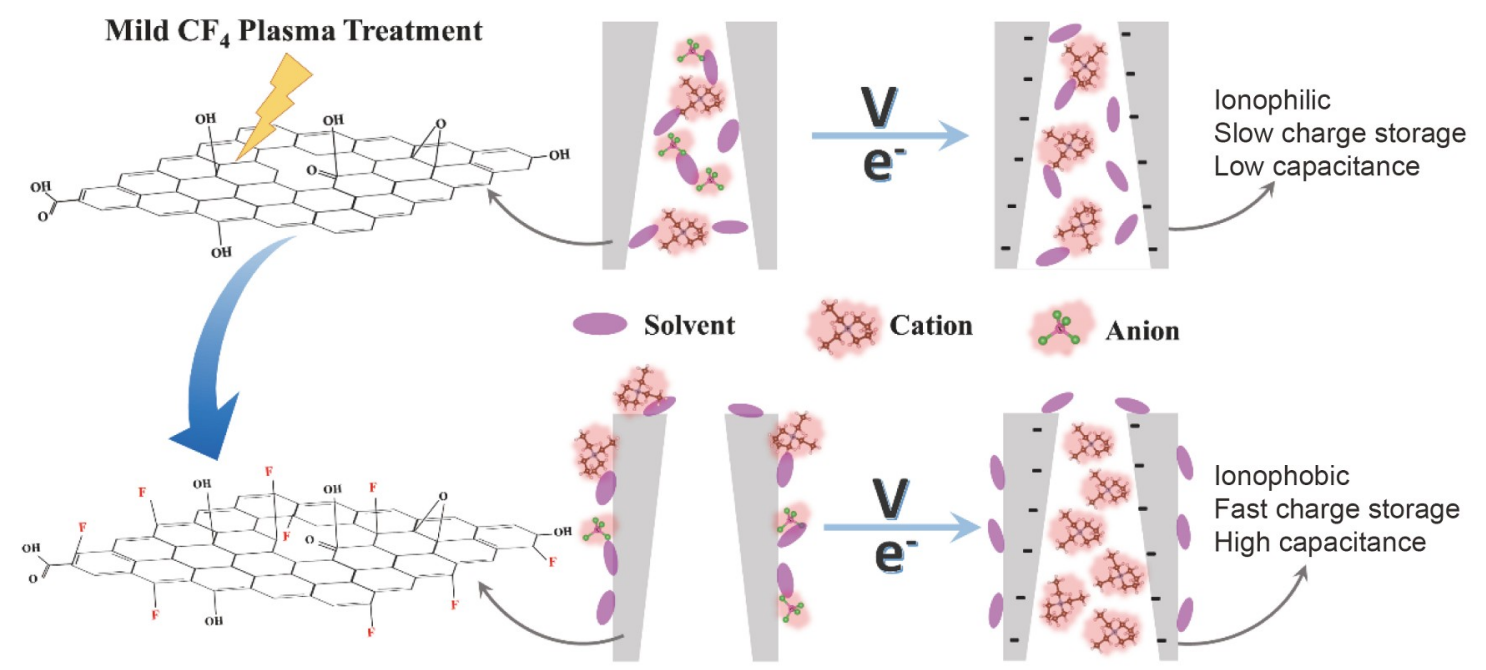

Scheme 1 Schematic illustration of a $\mathrm{CF}_{4}$ plasma treated carbon electrode with an ionophobic interfacial microenvironment in an organic electrolyte. 

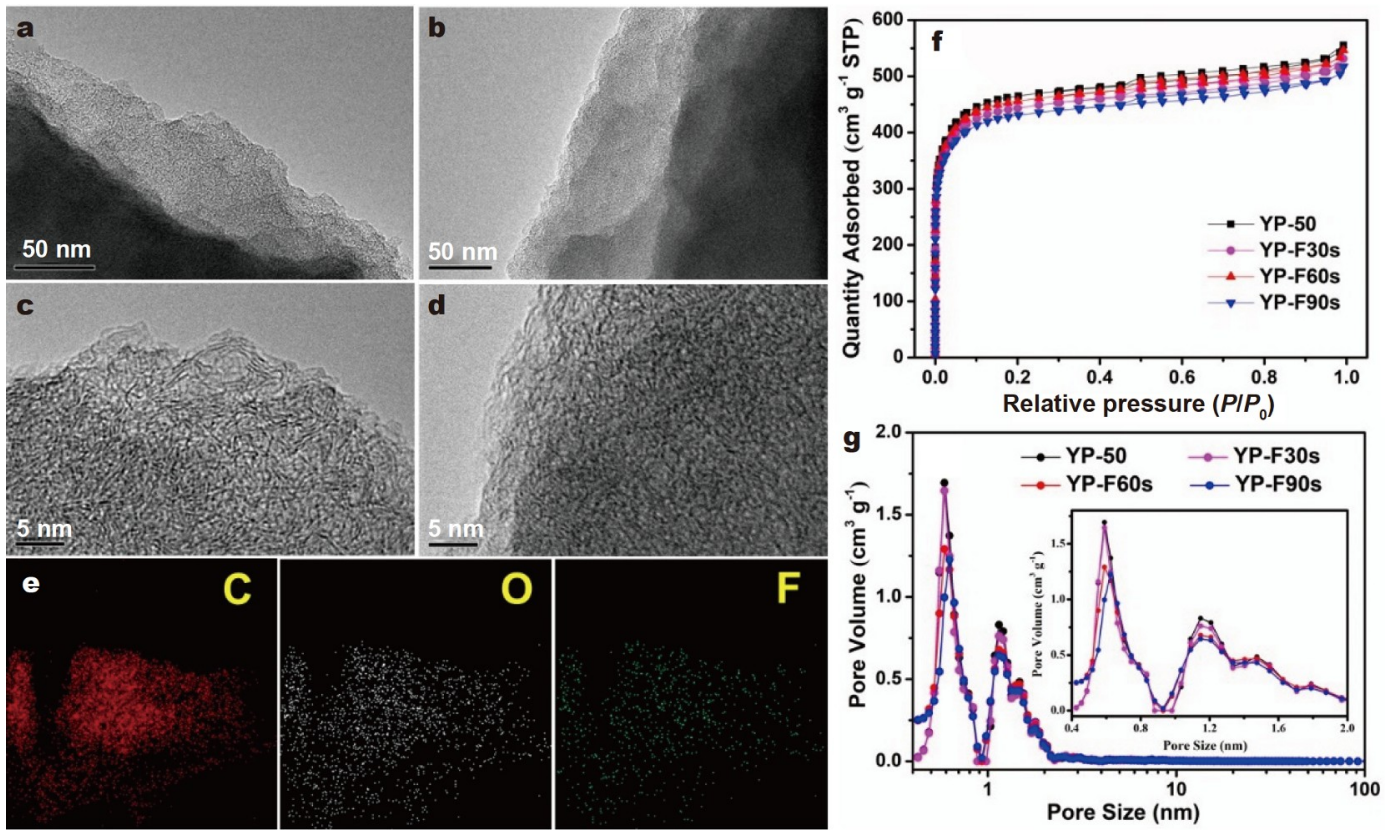

Figure 1 TEM images of (a) YP-50 and (b) YP-F60s. HRTEM images of (c) YP-50 and (d) YP-F60s. (e) EDX mapping images of YP-F60s. (f) Nitrogen adsorption-desorption isotherms and ( $\mathrm{g}$ ) pore-size distributions of the samples.

impact the morphology of the samples (Fig. S2). The HRTEM images obtained clearly show that YP-F60s maintains a disordered amorphous carbon structure, which indicates that plasma treatment does not destroy the morphology of the carbon material. The energy-dispersive X-ray spectroscopy (EDX) results suggest the uniform distribution of carbon (C), oxygen (O), and F atoms in YP-F60s (Fig. 1e). By comparison, YP-50 (Fig. S3) mainly consists of $\mathrm{C}$ and $\mathrm{O}$ atoms. These results indicate the successful incorporation of fluorine into YP-50 and its uniform dispersion on the surface of the material.

$\mathrm{N}_{2}$ adsorption-desorption measurements were carried out to further investigate the specific surface areas and pore-size distributions of the samples, as shown in Fig. 1f, g. Similar type I isotherms are observed for all samples, which confirms their microporous nature. Barret-Joyner-Halenda analysis shows that the pore-size distribution of YP-50 is mainly centered at $0.6,1.2$, and $1.5 \mathrm{~nm}$. Small micropores measuring $<2 \mathrm{~nm}$ in size are the dominant pore structure in the obtained microporous carbon. After $\mathrm{CF}_{4}$ plasma treatment, the pore-size distributions of YP-F do not change significantly, and the main pore structure of small micropores is retained. Microporous carbon materials with a small pore size could deliver superior capacitance in TEATFB/ PC electrolyte $[11,17,18]$. Table S1 summarizes the structural properties, specific surface areas, and the corresponding pore volumes of the samples, none of which appear to be affected by $\mathrm{CF}_{4}$ plasma treatment. The dominant contribution of small micropores to the total micropore volume of all samples could ensure a high charge storage capacity and rapid ion transport even under high current densities.

The XPS survey spectra of all samples are shown in Fig. 2a to investigate the effects of $\mathrm{CF}_{4}$ plasma treatment on the chemical structure of the AC. The XPS survey spectra of the YP-F samples show the peaks of $\mathrm{F} 1 \mathrm{~s}$, which are absent in the corresponding spectrum of pristine YP-50. The relative atomic contents of C decrease with increasing plasma treatment time in the order of
YP-50 > YP-F30s > YP-F60s > YP-F90s (Table S2). By contrast, the relative atomic contents of $\mathrm{F}$ increase, thereby indicating that $\mathrm{CF}_{4}$ plasma treatment results in the chemisorption of $\mathrm{F}$ atoms on the carbon surface. During the plasma discharge process, the $\mathrm{CF}_{4}$ gas medium is excited and dissociates to produce a large number of high-energy, active particles, including electrons, ions, and free radicals [19]. These energetic particles bombard the surface of the carbon material and cause physical and chemical reactions to occur. Fig. $2 \mathrm{~b}$ shows the $\mathrm{C} 1 \mathrm{~s}$ spectra of all samples. The peaks clearly shift toward lower binding energies after $\mathrm{CF}_{4}$ plasma treatment. These shifts are associated with the weakening of the $\mathrm{C}-\mathrm{C}$ bond due to the redistribution of the electron density in the carbon structure resulting from the interaction between the carbon matrix and $\mathrm{F}$ atoms. New peaks emerge in the energy range from 287 to $291 \mathrm{eV}$ because of the participation of photoelectrons emitted from $\mathrm{C}$ atoms in polar C-F bonding [20,21].

The chemical state of fluorine was identified through an analysis of the F 1s spectra (Fig. 2c). The asymmetric line of the $\mathrm{F}$ 1s core level signal reflects two types of polar C-F bonds. The signal at approximately $686.7 \mathrm{eV}$ corresponds to low-density fluorination, with individual $\mathrm{F}$ atoms semi-ionically bonded to $\mathrm{C}$ in the basal plane and characterized by a dilated $\mathrm{C}-\mathrm{F}$ bond. The second signal at approximately $688 \mathrm{eV}$ corresponds to the intense covalent $\mathrm{C}-\mathrm{F}$ bond, which results in a significant distortion of the carbon lattice [22]. Semi-ionic C-F bond contents progressively decrease, whereas covalent $\mathrm{C}-\mathrm{F}$ bond contents increase as the plasma treatment time increases. Increases in $\mathrm{F}$ doping content enhance the localization of $\pi$ electrons contributing to the $\mathrm{C}-\mathrm{F}$ bonds, resulting in a change in $\mathrm{C}-\mathrm{F}$ bond length and diminished ionic characteristics of the sample. Therefore, most C-F bonds tend to be formed as covalent bonds at high $\mathrm{F}$ doping contents.

The NEXAFS spectroscopy was used to probe the surface chemical functionalities and electronic structures of the samples. The NEXAFS C K-edge spectra of the samples reveal two main 

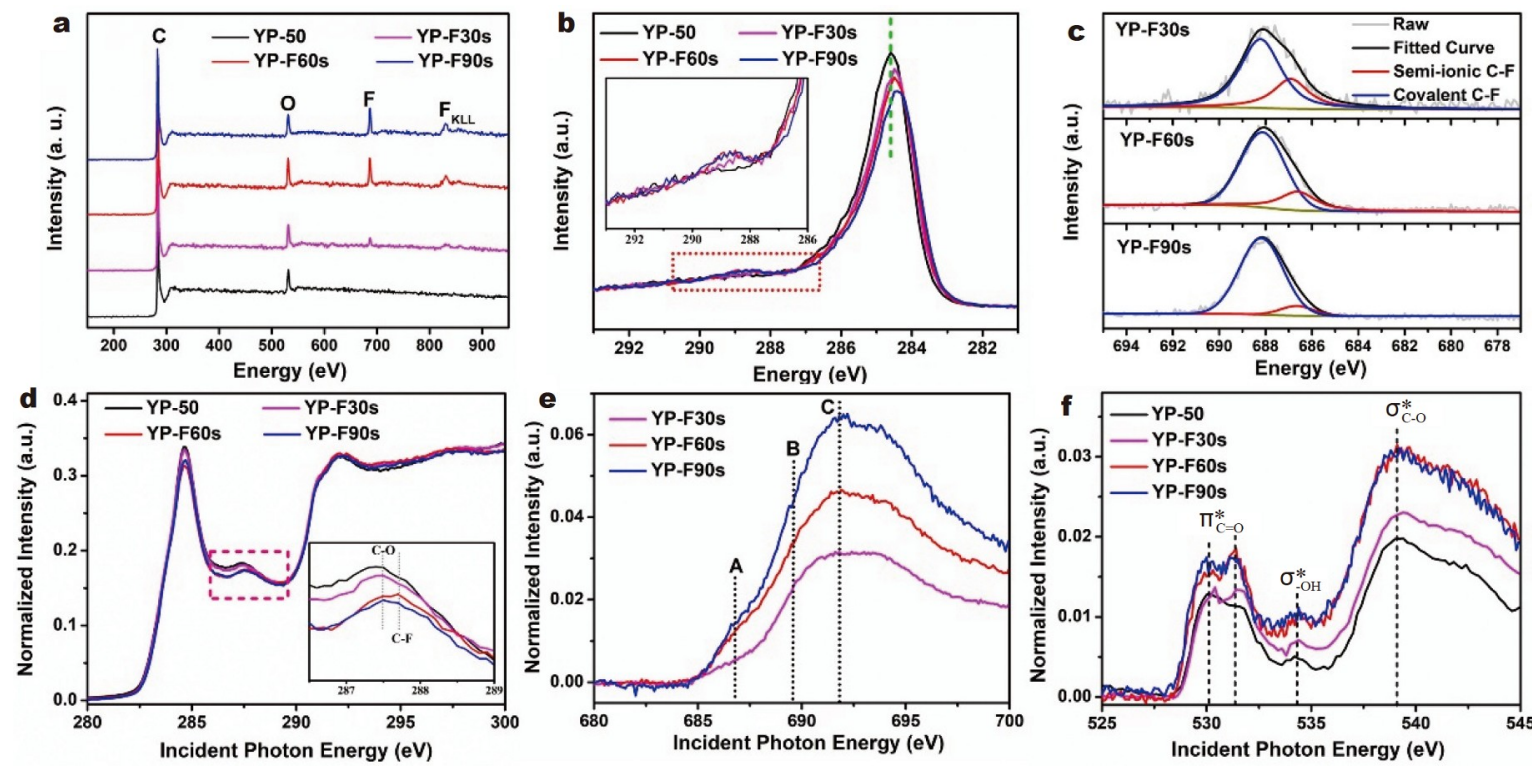

Figure 2 (a) XPS spectra of the surface chemical compositions of the samples. (b) XPS C 1s spectra of the samples. Inset: enlarged view of the area indicated by the dotted rectangle in the figure. (c) XPS F 1s spectra of the samples. (d-f) Carbon, nitrogen, and oxygen K-edge NEXAFS spectra of the samples.

resonances at 284.6 and $292.0 \mathrm{eV}$ (Fig. 2d), which correspond to the transitions of $1 \mathrm{~s} \rightarrow \pi^{*}$ and $1 \mathrm{~s} \rightarrow \sigma^{*}$, respectively [23]. The Kedge spectra of the plasma-treated samples show a reduction in $\pi^{*}$ resonance intensity and smoothing of the $\sigma^{*}$-resonance. These findings confirm the changes in the electronic structure of the carbon material due to $\mathrm{CF}_{4}$ plasma treatment. The relative intensity of the signals indicating the ratio of $\pi^{*}$ and $\sigma^{*}$ resonance intensities $\left(I_{\pi^{*}} / I_{\sigma^{*}}\right)$ decreases as the plasma time increases. This change indicates a decrease in graphitization degree, consistent with the Raman spectral results in Fig. S4. The features located between the $\pi^{*}$ and $\sigma^{*}$ resonance peaks refer to $\mathrm{C}$ atoms bound to $\mathrm{O}$ and $\mathrm{F}$; two peaks related to $\pi^{*}{ }_{\mathrm{C}-\mathrm{O}}(287.5 \mathrm{eV})$ and $\pi^{*}{ }_{\mathrm{C}-\mathrm{F}}(287.7 \mathrm{eV})$ may also be observed [24]. The spectra of the plasma-treated samples between 286 and $289 \mathrm{eV}$ differ from those of the pristine sample. Specifically, the characteristic peaks gradually shift toward higher energies as the plasma treatment time increases. These spectral changes indicate that $\mathrm{F}$ atoms are covalently attached to the surface of YP-F samples via breaks in the conjugated $\pi$ electron system.

The F K-edge NEXAFS spectra of the samples are shown in Fig. 2e, which illustrates broad peaks at $686.8 \mathrm{eV}$ (A peak), $689.6 \mathrm{eV}$ (B peak), and $692 \mathrm{eV}$ (C peak) [24-26]. These resonances correspond to excitations from the $\mathrm{F} 1 \mathrm{~s}$ state to the $\sigma^{*}$ state due to the covalent interaction between $\mathrm{F}$ and $\mathrm{C}$ atoms. According to the literature, peak $\mathrm{A}$ could be attributed to the four $\mathrm{F}$ atoms of $\mathrm{CF}_{4}$ forming a zigzag or armchair chain in the carbon structure. Peak B is attributed to the replacement of one edge $\mathrm{F}$ atom with $\mathrm{O}$-containing groups in the system of peak $\mathrm{A}$; these $\mathrm{O}$-containing functional groups belong to the pristine $\mathrm{YP}$ 50. Peak $\mathrm{C}$ is attributed to one $\mathrm{O}$ atom bonded to two fluorinated $\mathrm{C}$ atoms at the edge of a double atomic vacancy. As the plasma treatment time increases, the intensity of peak $\mathrm{A}$ increases significantly, which indicates that more $\mathrm{F}$ atoms are attached to the surface of carbon structures with conjugated $\pi$ electron systems. The intensity of peaks B and C also increase, thus suggesting that $\mathrm{F}$ atoms gradually interact with $\mathrm{O}$ atoms. Additional information on the surface chemistry between $\mathrm{O}$ and

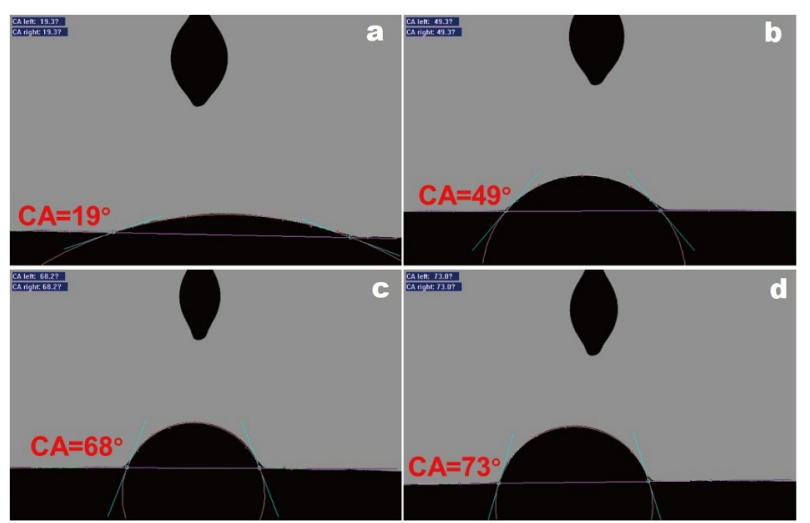

Figure 3 Wetting CA of electrolyte droplets left on the surfaces of (a) YP50, (b) YP-F30s, (c) YP-F60s, and (d) YP-F90s for $3 \mathrm{~s}$.

$\mathrm{F}$ atoms in the plasma-treated samples can be extracted from the $\mathrm{O}$ K-edge spectrum. As shown in Fig. 2f, the three absorption peaks of YP-50 at 530.1, 534.3, and 538-540 eV correspond to $\pi_{\mathrm{C}=\mathrm{O}}, \sigma^{*}{ }_{-\mathrm{OH}}$, and $\sigma^{*}{ }_{\mathrm{C}-\mathrm{O}}$, respectively [27]. After plasma treatment, the peaks of $\sigma^{*}{ }_{-\mathrm{OH}}$ and $\sigma^{*}{ }_{\mathrm{C}-\mathrm{O}}$ in the samples remain virtually unchanged. By contrast, the peak of $\pi^{*} \mathrm{C}=\mathrm{O}$ splits into two feature peaks related to $\mathrm{F}$ atoms. This finding indicates that most $\mathrm{F}$ atoms attach to non-functionalized carbon atoms rather than replace $\mathrm{O}$-contained groups.

Polar C-F bonds could change the wettability between the sample surface and electrolyte [16]. As shown in Fig. 3a-d, electrolyte droplets left on the sample surfaces for $3 \mathrm{~s}$ show distinct curvatures and different CAs. The CA of the pristine YP-50 is $19^{\circ}$, thereby demonstrating the ionophilic characteristic of this sample. The surface of YP-50 features a variety of Ocontaining groups that endow the material with ionophilic features. After $\mathrm{CF}_{4}$ plasma modification, the CAs of YP-F30s and YP-F60s are $49^{\circ}$ and $68^{\circ}$, respectively, which indicates a reduction in the surface energy of AC and a gradual increase in the ionophobic characteristics of the carbon surface. The CA further 
increases as the plasma duration is prolonged to $90 \mathrm{~s}$. When polar $\mathrm{C}-\mathrm{F}$ bonds are introduced to the carbon materials, their $\mathrm{CA}$ increases and their surface energy decreases. These effects cause changes in the ionophobic features of the sample surface $[11,14]$.

The SAXS spectroscopy was performed to illustrate the ionophobic properties of the plasma-modified AC, and SAXS curves were obtained from the pristine carbon materials or carbon materials soaked with $1 \mathrm{~mol} \mathrm{~L}^{-1}$ TEATFB/PC electrolyte. Fig. 4a shows the scattering vector lengths $Q$ ranging from 0.3 to $20 \mathrm{~nm}^{-1}$. Besides the SAXS signals (Q-range, $0.3-8 \mathrm{~nm}^{-1}$ ), wideangle X-ray scattering (WAXS) signals (Q-range, $9.5-20 \mathrm{~nm}^{-1}$ ), which were measured simultaneously with the SAXS signals by an additional detector, are displayed in the figure. The SAXS signals could be divided into three main regions according to the pores of different carbon structure levels. For example, the scattering vector modulus $Q$ occurs over a small area $(Q<$ $0.7 \mathrm{~nm}^{-1}$ ) and can be attributed to large pores (macropores) between the micron-sized carbon particles. The $Q$ in the intermediate region between 0.7 and $5 \mathrm{~nm}^{-1}$ is attributed to the scattering of disordered micropores. The Debye-AndersonBrumberger model is usually used to obtain the average correlation parameters related to the pore size and surface area in this region. When the $Q$ value is large $\left(Q>5 \mathrm{~nm}^{-1}\right)$, the molecular structural factors of the carbon matrix and electrolyte dominate, corresponding to the correlation between $\mathrm{C}$ atoms and electrolyte ions [18,28].

As shown in Fig. 4a, YP-50 is infiltrated with the electrolyte. The SAXS intensity of the medium- and low- $Q$ value regions is significantly reduced because of the reduction in electron density difference between the carbon structure and ion-filled pores. Compared with those of YP-F60s in the air or the carbon material soaked with $1 \mathrm{~mol} \mathrm{~L}^{-1}$ TEATFB/PC electrolyte, no difference in the intensity of SAXS in the low- and medium-Q value regions is noted. The above results indicate that the electrolyte ions penetrate the pores of YP-50. By contrast, the surface of YP-F60s prevents electrolyte ions from entering its pores because of its ionophobic characteristic. The WAXS intensity of YP-50 and YP-F60s soaked with TEATFB/PC electrolyte reflects the carbon structure factor (002)-peak (visible for the carbon material in the air) and the ion structure factor peak at approximately $15 \mathrm{~nm}^{-1}$ [28]. The strong WAXS intensity of YP50 may be due to the joint action of the molecular structures of the electrolyte and carbon, thus confirming that YP-F60s has ionophobic characteristics. The ionophobic feature of YP-F60s prevents electrolyte ions from entering the carbon nanopores in the absence of an applied voltage.

The scattering intensity of YP-F60s is high in the large- $Q$ value region (Fig. 4b) when the sample is immersed in $1 \mathrm{~mol} \mathrm{~L}^{-1}$ TEATFB/PC electrolyte and vacuumed overnight prior to measurement. This result indicates that ion-filled pores are formed in YP-F60s soaked with $1 \mathrm{~mol} \mathrm{~L}^{-1}$ TEATFB/PC when an external force (e.g., voltage) is applied to it.

The electrochemical performance of the YP-F samples was evaluated using symmetric two-electrode cells. Fig. 5a shows the $\mathrm{CV}$ plots of the cells at $10 \mathrm{mV} \mathrm{s}^{-1}$. The highly rectangular cyclic plots of all four materials show typical behavior of materials for EDLC. The CV curves of all samples at different scan rates of $10-100 \mathrm{mV} \mathrm{s}^{-1}$ are shown in Fig. S5. The current density increases linearly with increasing scan rate, thereby indicating that the reaction of all electrode materials at the electrode/ electrolyte interface is surface controlled. Fig. 5b shows the comparative CV plots of all samples at a scan rate of $100 \mathrm{mV} \mathrm{s}^{-1}$. Here the curves of the pristine YP-50 transform to a fusiform shape. By contrast, the $\mathrm{CF}_{4}$ plasma-treated YP-F mainly retains its rectangular shape. This phenomenon indicates that nanoporous YP-F has a stronger affinity toward electrolyte ions than YP-50 at a specific voltage and can efficiently form an electric double-layer within the nanopores of the carbon materials. The curves of YP-F60s most closely resemble a rectangle without significant distortion, thereby implying that YP-F60s exhibits superior rate capability and stability [29].

Fig. $5 \mathrm{c}$ shows the GCD curves of the samples at 0.5 and $10 \mathrm{~A} \mathrm{~g}^{-1}$. At a current density of $0.5 \mathrm{~A} \mathrm{~g}^{-1}$, the samples exhibit typical quasi-triangular and symmetric shapes, thereby indicating that they have excellent electrochemical reversibility and Coulombic efficiency. The symmetrical shape usually reflects ideal double-layer capacitance behavior. Among the samples tested, YP-F60s exhibits the longest discharge time, which corresponds to the highest specific capacitance. The specific capacitance of YP-F60s at a current density of $0.5 \mathrm{~A} \mathrm{~g}^{-1}$ is calculated to be $81.6 \mathrm{~F} \mathrm{~g}^{-1}$, which is higher than those of YP-50 $\left(62.2 \mathrm{~F} \mathrm{~g}^{-1}\right)$, YP-F30s $\left(76.9 \mathrm{Fg}^{-1}\right)$, and YP-F90s $\left(72.6 \mathrm{Fg}^{-1}\right)$. At a current density of $10 \mathrm{Ag}^{-1}$, YP-50 shows a significant voltage drop, thus suggesting insufficient charge-discharge reversibility. As the plasma treatment time increases, the voltage drop gradually decreases and the YP-F60s exhibits a minimum value. This result demonstrates that YP-F with ionophobic characteristics in the
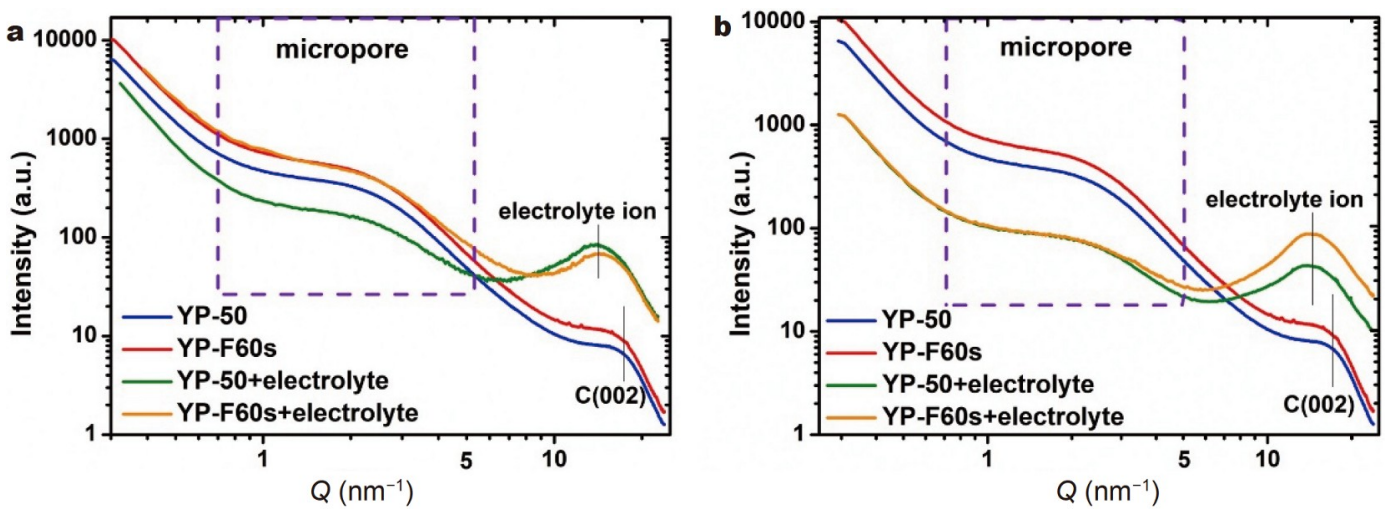

Figure 4 (a) SAXS curves of the pristine carbon electrodes in the air or soaked with $1 \mathrm{~mol} \mathrm{~L}^{-1} \mathrm{TEATFB} / \mathrm{PC}$ electrolyte. (b) SAXS curves of the pristine carbon electrodes in the air or soaked with $1 \mathrm{~mol} \mathrm{~L}^{-1}$ TEATFB/PC electrolyte after being vacuumed for $24 \mathrm{~h}$. 

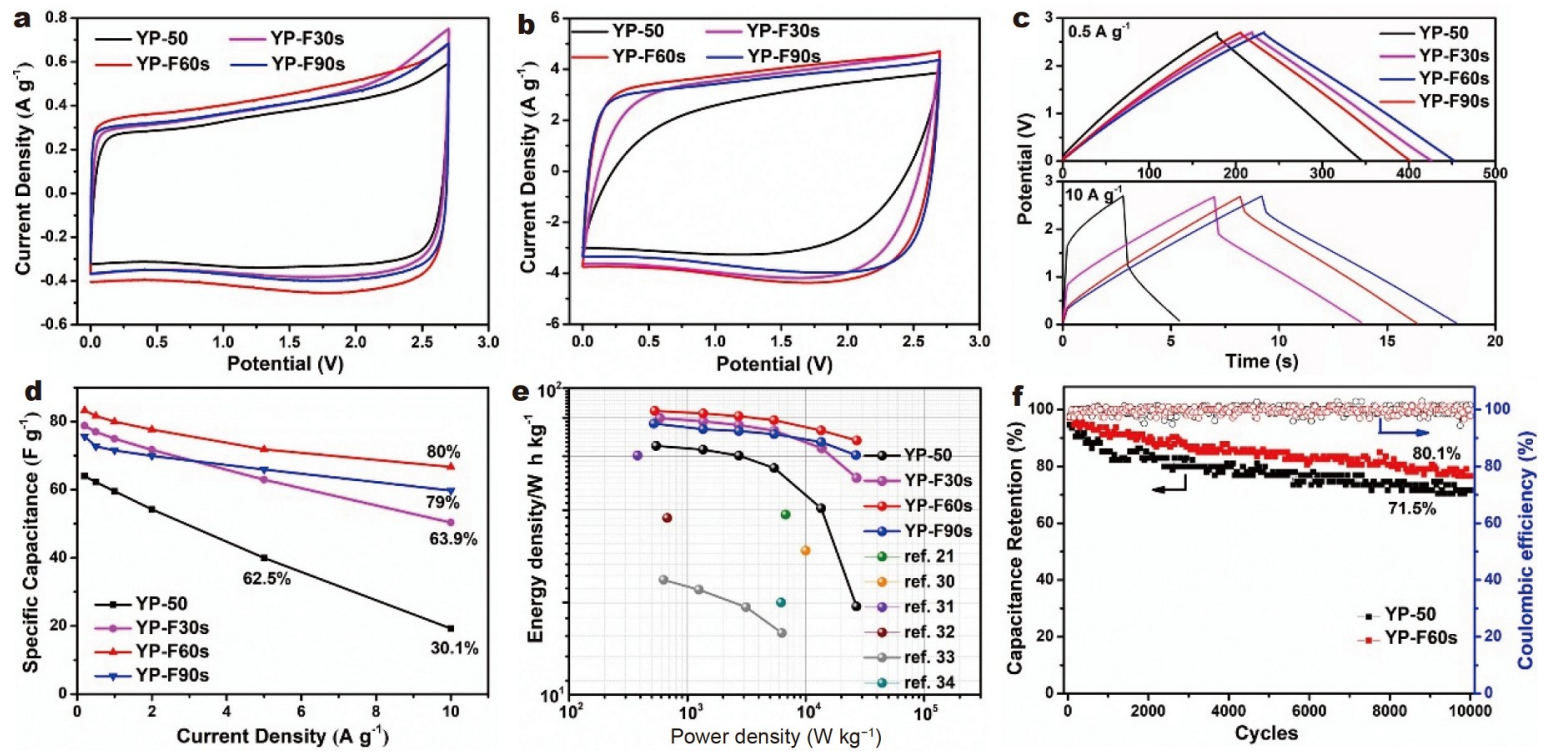

Figure $5 \mathrm{CV}$ curves of the samples measured in symmetrical two-electrode cells with $1 \mathrm{~mol} \mathrm{~L} \mathrm{~L}^{-1} \mathrm{TEATFB} / \mathrm{PC}$ electrolyte over the potential range of $0-2.7 \mathrm{~V}$ at scan rates of (a) $10 \mathrm{mV} \mathrm{s}^{-1}$ and (b) $100 \mathrm{mV} \mathrm{s}^{-1}$. (c) GCD curves obtained at 0.5 and $10 \mathrm{Ag}^{-1}$. (d) Capacitance retention of the samples at current densities ranging from 0.2 to $10 \mathrm{Ag}^{-1}$. (e) Ragone plots of the samples. (f) Cycling durability of the samples at a current density of $1 \mathrm{Ag}^{-1}$ over 10,000 cycles.

organic electrolyte can improve the electrochemical performance of carbon electrodes in EDLCs. The specific capacitance first increases and then decreases with increasing plasma treatment time, indicating that the ionophobic characteristics of the carbon electrode surface must be balanced at a specific voltage to optimize the affinity between the electrode and electrolyte ions. The GCD curves and specific capacitances of all samples at various current densities are shown in Fig. S6 and Table S3. The specific capacitances of all samples at different current densities are summarized and compared in Fig. $5 d$. The specific capacitance of all electrodes decreases slowly with increasing current density, which can be attributed to the short time available for the electrolyte ions to spread over the carbon surface after a voltage is applied to the electrode. The specific capacitance of the YP-F60s electrode at a current density of $0.2 \mathrm{~A} \mathrm{~g}^{-1}$ is $83.2 \mathrm{Fg}^{-1}$. Even at a high current density of $10 \mathrm{Ag}^{-1}$, high capacitance retention of up to $80 \%$ (i.e., $66.7 \mathrm{~F} \mathrm{~g}^{-1}$ ) may be obtained from this electrode. By comparison, the capacitance retention rates of YP50, YP-F30s, and YP-F90s are 30.1\%, $63.9 \%$, and 79\%, respectively. After $\mathrm{CF}_{4}$ plasma treatment, the specific capacitance and capacitance retention of the electrodes are remarkably improved because improvements in the interfacial microenvironment could promote rapid ion diffusion. YP-F60s shows excellent capacitance retention, which means its ionophobic characteristics can effectively enhance the adsorption-desorption capacity of electrolyte ions in the nanopores of the carbon electrode. The Ragone plots of all electrodes are displayed in Fig. 5e; here, the YP-F electrodes with ionophobic characteristics in the organic electrolyte show much higher energy densities than the pristine YP-50. The YP-F60s electrode offers a high energy density of $84.2 \mathrm{Wh} \mathrm{kg}^{-1}$ at a power density of $528.7 \mathrm{~W} \mathrm{~kg}^{-1}$; the energy density obtained from this electrode is higher than the values reported for similar devices [21,30-34].

The cycling life of the cells was tested by GCD measurements at $1 \mathrm{~A} \mathrm{~g}^{-1}$ and $2.7 \mathrm{~V}$ to further evaluate the durability of the YP50 and YP-F electrodes. As shown in Fig. 5f, the cycling dur- ability of the YP-F60s electrode is much greater than that of the YP-50 electrode over 10,000 cycles, clearly indicating the excellent cycling durability of the YP-F60s electrode with the capacitance retention of $80.2 \%$. The Coulombic efficiencies obtained are near $100 \%$, confirming that YP-F60s has excellent electrochemical reversibility. Thus, YP-F treated with $\mathrm{CF}_{4}$ plasma exhibits enhanced electrochemical reaction stability and high Coulombic efficiency (Fig. S7). The excellent cycling stability of YP-F60s can be ascribed to its ionophobicity, which inhibits electrolyte degradation. The introduction of $\mathrm{F}$ atoms also increases repulsive interactions on the carbon lattice so that the $\mathrm{F}$ atoms are more stable at the edges of the lattice than in the interior region of the material [20]. This propensity renders the edge position of the carbon lattice in YP-F60s more durable and resistant to carbon corrosion during charging/discharging.

The EIS was conducted to investigate the materials' charge transfer and conductivity. Nyquist plots of the electrodes are presented in Fig. 6a. All sample plots feature an incomplete semicircle in the high-frequency range and a non-straight line in the low-frequency region. The semicircle diameter represents the charge-transfer resistance $\left(R_{\mathrm{ct}}\right)$ at the electrode/electrolyte interface originating from the double-layer capacitance on the samples' surface. The slope of the line corresponds to the capacitive behavior and ion diffusion resistance of the samples. Compared with YP-50, the YP-F electrodes exhibit a smaller ohmic resistance and $R_{\mathrm{ct}}$, consistent with the conductivity of carbon electrodes (Fig. S8). This result indicates that the ionophobic characteristics of the carbon electrode surface can promote electron/ion transfer in the electrode/electrolyte interface. Among the samples tested, YP-F60s shows the lowest $R_{\text {ct }}$. Moreover, the $R_{\mathrm{ct}}$ values of YP-F90s increase with increasing plasma treatment time, thereby indicating that excessive ionophobicity reduces the affinity between the carbon electrode and electrolyte ions under an applied potential, which increases the ion diffusion resistance. The slope of the straight lines in the low-frequency regions increases remarkably after $\mathrm{CF}_{4}$ plasma 


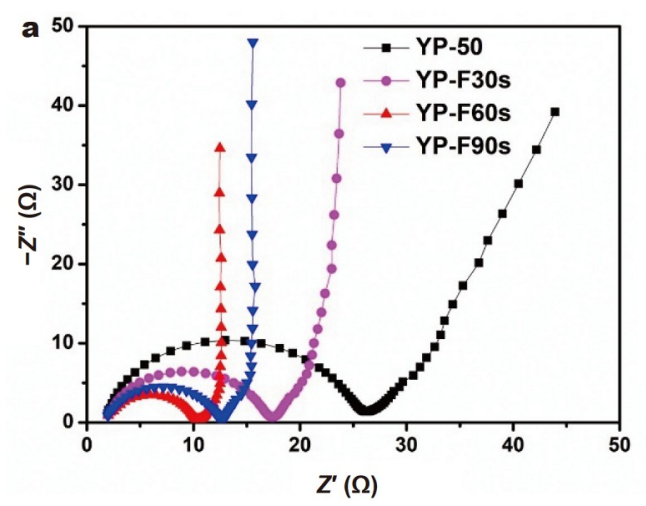

Figure 6 (a) Nyquist and (b) Bode plots of all samples.

treatment, thereby indicating that carbon electrodes with ionophobic characteristics are beneficial to forming an electric double-layer at a specific voltage. Fig. $6 \mathrm{~b}$ shows the Bode plots of the samples. Here, the phase angle at the low-frequency region increases noticeably after plasma treatment, thus revealing that the existence of ionophobic characteristics enhances the ion migration. The phase angle of YP-F60 is $-72^{\circ}$, which is close to the angle necessary to achieve ideal EDLC behavior and effectively reduces the ion diffusion resistance. This finding is supported by the high capacitance retention of the samples at high current densities.

\section{CONCLUSION}

In summary, we developed a novel and facile approach to adjust the interfacial microenvironment between a carbon electrode and an electrolyte. YP-50 was treated with $\mathrm{CF}_{4}$ plasma to form $\mathrm{C}-\mathrm{F}$ bonds. These bonds modified the microenvironment of YP50 and its confined organic electrolyte. The ionophobic characteristics of the carbon electrode in $1 \mathrm{~mol} \mathrm{~L}^{-1}$ TEATFB/PC were regulated by adjusting the treatment time. Compared with the pristine YP-50, YP-F60s revealed remarkably higher specific capacitance in symmetric cells with lower internal resistance and excellent retention at high charge/discharge rates and prolonged cycling times. The present study demonstrates a simple and effective strategy to increase the double-layer capacitance of an electrode by altering its microenvironment. The results of this strategy could be used to obtain novel insights into the storage mechanisms of EDLCs.

Received 18 April 2021; accepted 29 June 2021; published online 30 August 2021

1 Shao $\mathrm{H}$, Wu YC, Lin Z, et al. Nanoporous carbon for electrochemical capacitive energy storage. Chem Soc Rev, 2020, 49: 3005-3039

2 Yan J, Li S, Lan B, et al. Rational design of nanostructured electrode materials toward multifunctional supercapacitors. Adv Funct Mater, 2020, 30: 1902564

3 González A, Goikolea E, Barrena JA, et al. Review on supercapacitors: Technologies and materials. Renew Sustain Energy Rev, 2016, 58: 1189_ 1206

4 Raza W, Ali F, Raza N, et al. Recent advancements in supercapacitor technology. Nano Energy, 2018, 52: 441-473

5 Yang Z, Tian J, Yin Z, et al. Carbon nanotube- and graphene-based nanomaterials and applications in high-voltage supercapacitor: A review. Carbon, 2019, 141: 467-480

6 Xie L, Su F, Xie L, et al. Effect of pore structure and doping species on charge storage mechanisms in porous carbon-based supercapacitors.

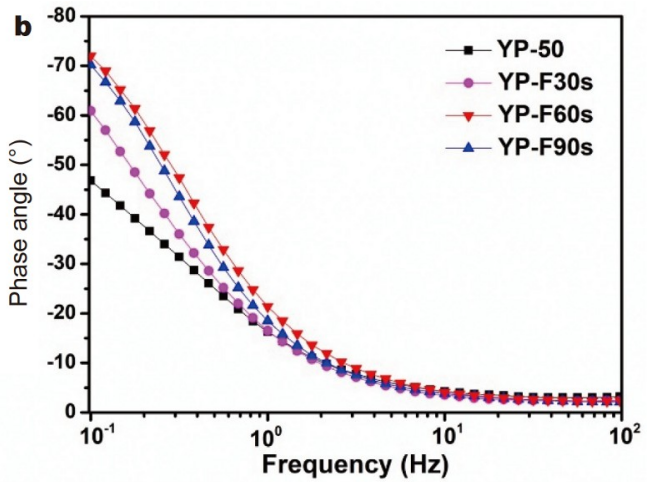

Mater Chem Front, 2020, 4: 2610-2634

7 Qi D, Liu Y, Liu Z, et al. Design of architectures and materials in inplane micro-supercapacitors: Current status and future challenges. Adv Mater, 2017, 29: 1602802

8 Simon P, Gogotsi Y. Perspectives for electrochemical capacitors and related devices. Nat Mater, 2020, 19: 1151-1163

9 Eliad L, Salitra G, Soffer A, et al. Ion sieving effects in the electrical double layer of porous carbon electrodes: Estimating effective ion size in electrolytic solutions. J Phys Chem B, 2001, 105: 6880-6887

10 Eliad L, Salitra G, Soffer A, et al. On the mechanism of selective electroadsorption of protons in the pores of carbon molecular sieves. Langmuir, 2005, 21: 3198-3202

11 Forse AC, Merlet C, Griffin JM, et al. New perspectives on the charging mechanisms of supercapacitors. J Am Chem Soc, 2016, 138: 5731-5744

12 Redondo E, Tsai WY, Daffos B, et al. Outstanding room-temperature capacitance of biomass-derived microporous carbons in ionic liquid electrolyte. Electrochem Commun, 2017, 79: 5-8

13 Griffin JM, Forse AC, Tsai WY, et al. In situ NMR and electrochemical quartz crystal microbalance techniques reveal the structure of the electrical double layer in supercapacitors. Nat Mater, 2015, 14: 812-819

14 Kondrat S, Kornyshev AA. Pressing a spring: What does it take to maximize the energy storage in nanoporous supercapacitors? Nanoscale Horiz, 2016, 1: 45-52

15 Kondrat S, Wu P, Qiao R, et al. Accelerating charging dynamics in subnanometre pores. Nat Mater, 2014, 13: 387-393

16 Qian C, Zhao J, Sun Y, et al. Electrolyte-phobic surface for the nextgeneration nanostructured battery electrodes. Nano Lett, 2020, 20: 7455-7462

17 Salanne M, Rotenberg B, Naoi K, et al. Efficient storage mechanisms for building better supercapacitors. Nat Energy, 2016, 1: 16070

18 Prehal C, Koczwara C, Jäckel N, et al. Quantification of ion confinement and desolvation in nanoporous carbon supercapacitors with modelling and in situ X-ray scattering. Nat Energy, 2017, 2: 16215

$19 \mathrm{Lu} \mathrm{M}, \mathrm{He} \mathrm{Q}, \mathrm{Li} \mathrm{Y}$, et al. The effects of radio-frequency $\mathrm{CF}_{4}$ plasma on adhesion properties of vertically aligned carbon nanotube arrays. Carbon, 2019, 142: 592-598

20 Bahuguna G, Chaudhary S, Sharma RK, et al. Electrophilic fluorination of graphitic carbon for enhancement in electric double-layer capacitance. Energy Technol, 2019, 7: 1900667

$21 \mathrm{Na}$ W, Jun J, Park JW, et al. Highly porous carbon nanofibers co-doped with fluorine and nitrogen for outstanding supercapacitor performance. J Mater Chem A, 2017, 5: 17379-17387

22 Zhao $\mathrm{Y}$, Wei $\mathrm{K}, \mathrm{Wu} \mathrm{H}$, et al. LiF splitting catalyzed by dual metal nanodomains for an efficient fluoride conversion cathode. ACS Nano, 2019, 13: acsnano.8b09453

23 Yin $\mathrm{X}$, Utetiwabo $\mathrm{W}$, Sun $\mathrm{S}$, et al. Incorporation of $\mathrm{CeF}_{3}$ on single-atom dispersed $\mathrm{Fe} / \mathrm{N} / \mathrm{C}$ with oxophilic interface as highly durable electrocatalyst for proton exchange membrane fuel cell. J Catal, 2019, 374: 4350

24 Fedoseeva YV, Bulusheva LG, Koroteev VO, et al. Preferred attachment 
of fluorine near oxygen-containing groups on the surface of doublewalled carbon nanotubes. Appl Surf Sci, 2020, 504: 144357

25 Bulusheva LG, Fedoseeva YV, Flahaut E, et al. Effect of the fluorination technique on the surface-fluorination patterning of double-walled carbon nanotubes. Beilstein J Nanotechnol, 2017, 8: 1688-1698

26 Struzzi C, Scardamaglia M, Colomer JF, et al. Fluorination of vertically aligned carbon nanotubes: From $\mathrm{CF}_{4}$ plasma chemistry to surface functionalization. Beilstein J Nanotechnol, 2017, 8: 1723-1733

27 Hemraj-Benny T, Banerjee S, Sambasivan S, et al. Near-edge X-ray absorption fine structure spectroscopy as a tool for investigating nanomaterials. Small, 2006, 2: 26-35

28 Prehal C, Weingarth D, Perre E, et al. Tracking the structural arrangement of ions in carbon supercapacitor nanopores using in situ small-angle X-ray scattering. Energy Environ Sci, 2015, 8: 1725-1735

29 Zhou J, Xu L, Li L, et al. Polytetrafluoroethylene-assisted N/F co-doped hierarchically porous carbon as a high performance electrode for supercapacitors. J Colloid Interface Sci, 2019, 545: 25-34

30 Vijayakumar M, Santhosh R, Adduru J, et al. Activated carbon fibres as high performance supercapacitor electrodes with commercial level mass loading. Carbon, 2018, 140: 465-476

31 Zhang S, Shi X, Wróbel R, et al. Low-cost nitrogen-doped activated carbon prepared by polyethylenimine (PEI) with a convenient method for supercapacitor application. Electrochim Acta, 2019, 294: 183-191

32 Wang D, Liu S, Jiao L, et al. Unconventional mesopore carbon nanomesh prepared through explosion-assisted activation approach: A robust electrode material for ultrafast organic electrolyte supercapacitors. Carbon, 2017, 119: 30-39

33 Yang V, Arumugam Senthil R, Pan J, et al. Hierarchical porous carbon derived from jujube fruits as sustainable and ultrahigh capacitance material for advanced supercapacitors. J Colloid Interface Sci, 2020, 579: $347-356$

34 Kota M, Jana M, Park HS. Improving energy density of supercapacitors using heteroatom-incorporated three-dimensional macro-porous graphene electrodes and organic electrolytes. J Power Sources, 2018, 399: $83-88$

Acknowledgements This work was supported by the National Natural Science Foundation of China (21203008 and 21975025), Beijing Natural Science Foundation (2172051) and the State Key Laboratory for Modification of Chemical Fibers and Polymer Materials, Donghua University. We appreciate the help from Dr. Jiaou Wang (4B9B@Beijing Synchrotron Radiation Facility) for XANES measurement. XPS measurements were performed at the Analysis \& Testing Center, Beijing Institute of Technology.

Author contributions Yang W conceived and supervised the project. Yin $\mathrm{X}$ and Yang $\mathrm{W}$ designed the experiment, carried out the material characterization, electrochemical measurements, analyzed the data and wrote the paper. Zhang J and Tang Y performed the SAXS analysis. Yang L and Zhou L performed the data analysis. Xiao W contributed to the theoretical analysis. All authors contributed to the general discussion and approved the final version of the manuscript.

Conflict of interest The authors declare that they have no conflict of interest.

Supplementary information version of the paper.

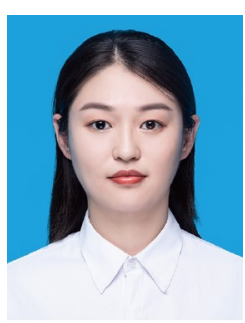

Xue Yin is currently a $\mathrm{PhD}$ candidate at the School of Chemistry and Chemical Engineering, Beijing Institute of Technology, under the supervision of Dr. Wen Yang. Her interest mainly focuses on nanostructured materials for energy storage and transformation devices, including electrochemical capacitors and proton-exchange membrane fuel cells.

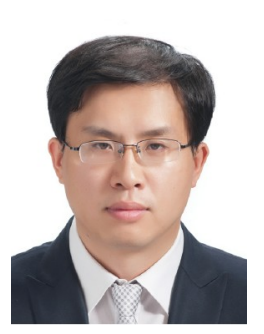

Wen Yang received his $\mathrm{PhD}$ degree from Changchun Institute of Applied Chemistry, Chinese Academy of Sciences, in 2003. Following a brief stint at the Max Plank Institute of Colloids and Interfaces, he joined Beijing Institute of Technology in 2011. In 2016, he was promoted to associate professor. His research interests focus on energy storage and transformation devices, including lithium-ion power batteries and materials, solid-state electrolytes, electrochemical capacitors, and proton-exchange membrane fuel cells.

\section{具有疏离子特性的碳电极用于高性能有机电解液双 电层电容器}

印雪 ${ }^{1}$, 张建齐 ${ }^{2}$, 杨乐 ${ }^{1}$, 肖文德 ${ }^{3}$, 周雷 ${ }^{1}$, 唐毓婧 ${ }^{4}$, 杨文 $^{1^{*}}$

摘要 活性碳材料应用于有机电解液双电层电容器中存在比电容低的 问题, 但目前的研究主要围绕改善活性碳的比表面积和孔结构来提高 双电层电容器的性能, 而活性碳纳米孔与有机电解液形成的界面微环 境往往被忽略. 本工作通过一个简单而有效的方法形成具有疏离子表 面的活性碳以解决双电层过程低效率的问题. 在活性碳上形成极性的 $\mathrm{C}-\mathrm{F}$ 键, 并用近边X射线吸收精细结构光谱和X射线光电子能谱对其进 行了表征. 通过接触角和小角X射线散射研究发现YP-F60s在有机电解 液中具有疏离子特性. 采用YP-F60s制备的双电层电容器在有机电解液 中展现出高比电容, 较低的内阻和优良的循环稳定性. 本论文证明了活 性碳与电解液形成的界面微环境对双电层电容器电化学性能的重要 性, 也为 $\mathrm{CF}_{4}$ 等离子体技术构建低成本、优质的碳材料用于双电层电容 器提供了新的思路. 